\title{
Effect of the Absorption on the Shape of the Emitted $\phi(\rho z)$ Depth Distribution for Accurate Quantitative Microanalysis: Evaluation of Analytical Models and Monte Carlo Programs
}

\author{
Hendrix Demers ${ }^{1}$, Meiken Falke ${ }^{2}$, Ralf Terborg ${ }^{2}$ and Raynald Gauvin ${ }^{1}$ \\ 1. Department of Mining and Materials Engineering, McGill University, Montreal, Quebec, Canada. \\ 2. Bruker Nano GmbH, Berlin, Germany.
}

In quantitative microanalysis, the correction factors are typically calculated using analytical models $[1,2]$. The $\phi(\rho z)$ models are the most accurate correction program available. Their excellent performances are achieved by calculating a realistic $\phi(\rho z)$ depth distribution. These models were largely developed and verified using experimental measurements. However, Monte Carlo simulations were used to complement missing or inaccessible experimental data. Previously, experimental measurements of $\phi(\rho z)$ depth distribution were used to evaluate the $\mathrm{x}$-ray generation models used in $\phi(\rho z)$ analytical models and Monte Carlo programs. Each model or program predict a different shape of the $\phi(\rho z)$ depth distribution. In this work, these different shapes are evaluated for three absorption cases: no absorption, moderate absorption and strong absorption.

In Figure 1, the experimental generated (without absorption) $\phi(\rho z)$ of aluminium (Al) $\mathrm{K} \alpha$ for two tracer thicknesses was compared to the calculated $\phi(\rho z)$ using XPP [1] and PROZA96 [2] analytical models and the simulated $\phi(\rho z)$ obtained with MC X-Ray [5] Monte Carlo program for accelerating voltage of 10 and $12 \mathrm{kV}$. The experimental $\phi(\rho z)$ was affected by the tracer thicknesses. A good agreement with the calculated $\phi(\rho z)$ was obtained for the thicker tracer film. However, the shape of the $\phi(\rho z)$ obtained by the three models and program was different. No agreement on the correct shape of the $\phi(\rho z)$ depth distribution can be found between the analytical model, Monte Carlo simulations and experimental measurements for the same specimen at different energies or for different specimens at the same energy.

Figure 2 shows the effect of the absorption on the shape of the emitted (with absorption) $\phi(\rho z)$ depth distribution in copper for three cases of absorption. In the first case, the sample does not absorb the $\mathrm{Cu}$ $\mathrm{K}_{\alpha} \mathrm{x}$-ray line (Fig. 2 left), i.e., the generated and emitted $\phi(\rho z)$ have the same shape. The second case shows that the $\mathrm{Cu} \mathrm{L}_{\alpha} \mathrm{x}$-ray line was absorbed by the sample, but the shape of emitted and generated $\phi(\rho z)$ are similar (Fig. 2 center). Only the amount of x-ray (area under the curve) was different. In the last case, the $\mathrm{Cu} \mathrm{L} \mathrm{L}_{\alpha} \mathrm{X}$-ray line was strongly absorbed by the sample and the shape of the emitted $\phi(\rho z)$ was completely different than the generated. In this case, the emitted $\phi(\rho z)$ shape was an exponential decay function and the accuracy of the generated $\phi(\rho z)$ calculated by the models or programs was not important. However, the accuracy of the models and programs depend on the mass absorption coefficient and the $\phi(0)$ value used.

A systematic study of the $\phi(\rho z)$ depth distribution obtained with analytical models and Monte Carlo programs are on-going for a large range of elements and binary alloys in the SEM accelerating voltage range $(0.5 \mathrm{kV}$ to $30 \mathrm{kV})$ with a focus on light element and low accelerating voltage. Comparison with the available experimental measurements is included in this study. 


\section{References:}

[1] J. L. Pouchou and F. Pichoir, in: Electron Probe Quantitation, Plenum Press (1991), pp. 31-75.

[2] G. F. Bastin et al., X-ray Spectrometry, 27 (1998), pp. 3-10.

[3] P. Karduck and W. Rehbach, in: Electron Probe Quantitation, Plenum Press (1991), pp. 191-217.

[4] J. D. Brown and L. Parobek, in: $6^{\text {th }}$ ICXOM, University of Tokyo Press, (1972), p. 163.

[5] R. Gauvin and P. Michaud, Microscopy and Microanalysis, 15 (2009), pp. 488-489.

[6] P. Hovington et al., Scanning 19 (1997), pp. 1-14; D. Drouin et al., Scanning 29 (2007), pp. 92-101.
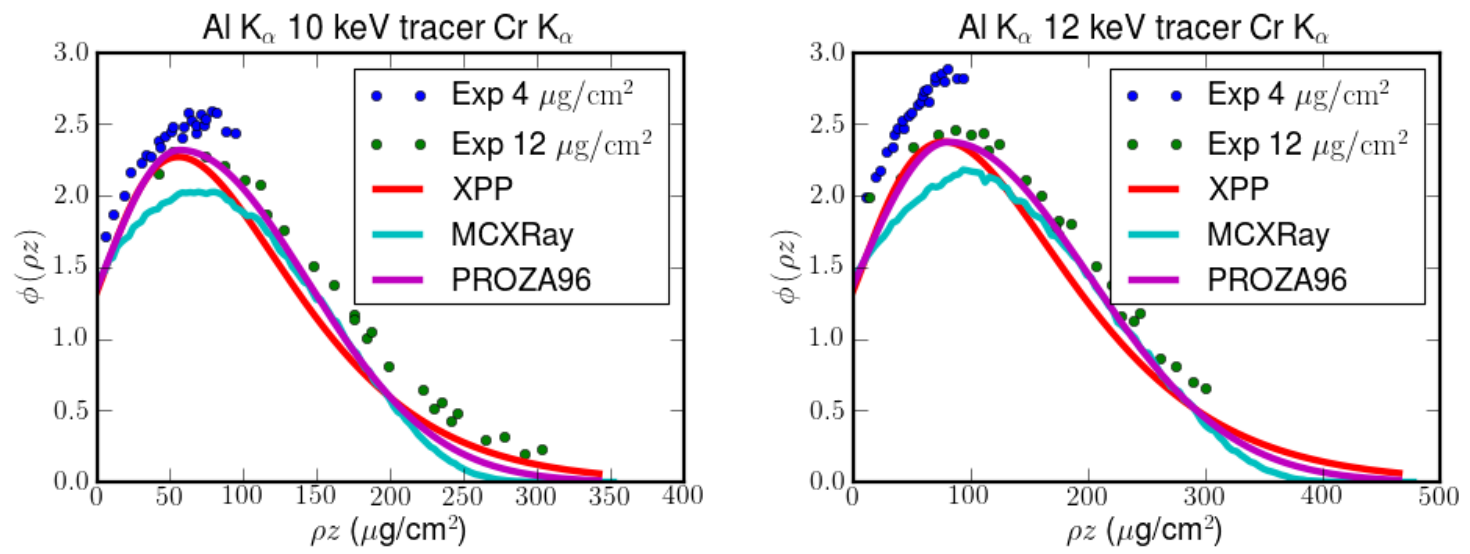

Figure 1 Thin film thickness effect. Comparison of $\phi(\rho z)$ depth distribution of aluminium (Al) $\mathrm{K}_{\alpha}$ at 10 and $12 \mathrm{keV}$ : experimental measurements of Brown and Parobek [3,4] (dot), XAP analytical model [1] (red line), MC X-Ray [5] (cyan line) and PROZA96 [2] (pink line).
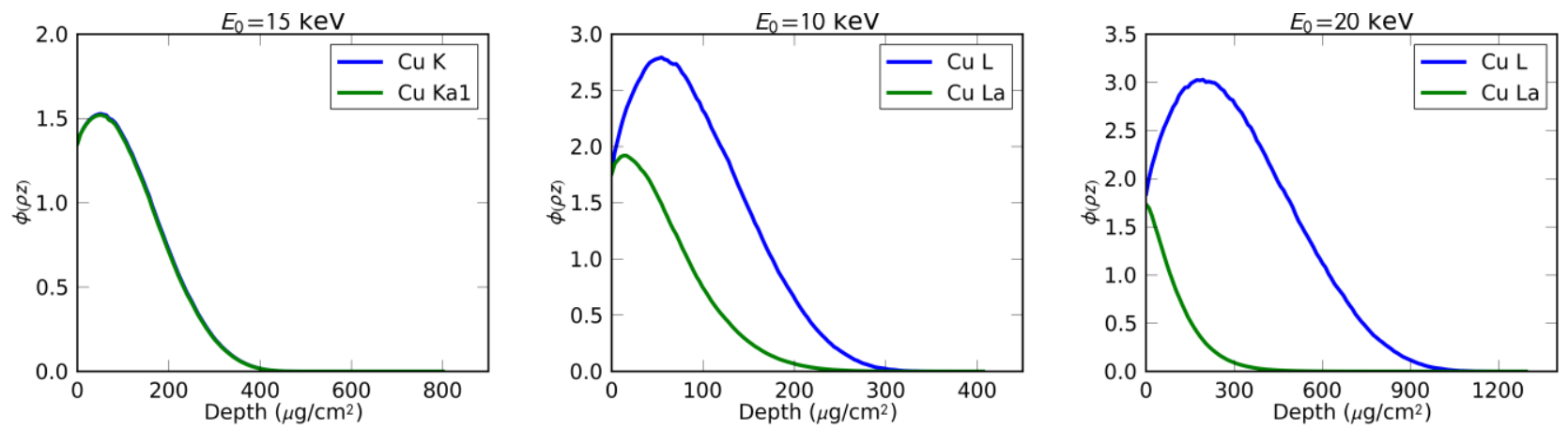

Figure 2 Effect of the absorption on the shape of the $\phi(\rho z)$ depth distribution in bulk copper obtained with MC X-Ray [5]. Left: no absorption for the $\mathrm{Cu} \mathrm{K}_{\alpha}$ X-ray line at $15 \mathrm{keV}$. Center: moderate absorption for the $\mathrm{Cu} \mathrm{L}_{\alpha} \mathrm{X}$-ray line at $10 \mathrm{keV}$. Right: strong absorption for the $\mathrm{Cu} \mathrm{L}_{\alpha} \mathrm{X}$-ray line at $20 \mathrm{keV}$. The shape of the generated $\phi(\rho z)$ depth distribution (blue line) does not change significantly. The shape of the emitted $\phi(\rho z)$ depth distribution (green line) change strongly with an increase of the absorption effect. 